\title{
Data Dictionary Classification using Multilayer Artificial Neural Network with New Error Metrics
}

\author{
Jitendra Nath Shrivastava \\ Singhania University, \\ Jhunjhunu, Pacheri Bari, \\ Rajasthan, India
}

\author{
Maringanti Hima Bindu \\ Department of Computer Science and Applications, \\ North Orissa University, India
}

\begin{abstract}
The ANN technique is inspired by biological neurons. In past, Artificial Neural Networks has been used as a data classification technique. In this paper, artificial neural network is used as a data classifier. Here, new error metric are considered in the data classification. The results presented in the paper, clearly shows that ANN can acts as a very good classifier with new error metrics.
\end{abstract}

Keywords: Data Dictionary; ANN; LMS; geometric error metric, Harmonic error metric.

\section{INTRODUCTION}

In many applications, data classification is very necessary. In past Artificial neural network has been used as data classifier with accuracy more than 95\%.[1,2] However, in some application the accuracy level of more than $99 \%$ is required. One such application is spam e-mail filtering where, data dictionary which consists of spam words need to be very accurate.

A neural network is an interconnected group of artificial neurons that uses a mathematical or computational model for information processing based on a connectionist approach [3]. Artificial neural networks are parallel computational models which are able to map any nonlinear functional relationship between an input and an output hyperspace to desired accuracy. They are constituted by individual processing units called neurons or nodes and differ among each other in the way these units are connected to process the information and, consequently in the kind of learning protocol adopted [4].

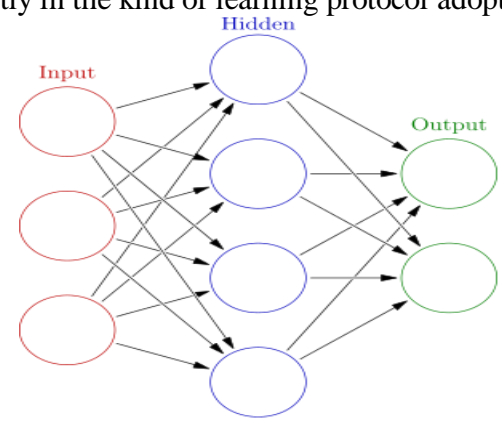

Figure 1 The Basic ANN Structure

In particular, the neurons of a feed-forward neural network are organized in three layers: the input units receive information from the outside world, usually in the form of a data file; the intermediate neurons, contained in one or more hidden layers, allow nonlinearity in the data processing, the output layer is used to provide an answer for a given set of input values [Figure 1]. In a fully connected artificial neural network, each neuron in a given layer is connected to each neuron in the following layer by an associated numerical weight ( $w_{i j}$ ), the weight that passes between them. In addition, each neuron possesses a numerical bias term corresponding to an input of -1 whose associated weight has the meaning of a threshold value. Rumelhart et al. [5] popularized the use of backpropagation for learning internal representation in neural networks. Back-propagation (BP) algorithm is the most widely used search technique for training neural networks. Information in an ANN is stored in the connection weights which can be thought of as the memory of the system. The purpose of BP training is to change iteratively the weights between the neurons in a direction that minimizes the error $\mathrm{E}$, defined as the squared difference between the desired and the actual outcomes of the output nodes, summed over training patterns (training set data) and the output neurons. The algorithm uses a sample-by-sample updating rule for adjusting connection weights in the network. In one algorithm iteration, a training sample is presented to the network. The signal is then fed in a forward manner through the network until the network output is obtained. The error between the actual and desired network outputs is calculated and used to adjust the connection weights.

Basically, the adjustment procedure, derived from a gradient descent method, is used to reduce the error magnitude. The procedure is firstly applied to the connection weights in the output layer, followed by the connection weights in the hidden layer next to output layer. This adjustment is continued backward through to network until connection weights in the first hidden layer are reached. The iteration is completed after all connection weights in the network have been adjusted. In this study, training of the multi-layer neural networks is implemented with back-propagation algorithm and network structure that has been trained with back-propagation algorithm has been used in the solutions of the multi-group classification models.

In Back-propagation algorithm, training of the neuron model is done by minimizing the error between target value and the observed value. In order to determine error between target and observed value, distance metric is used. It has been observed that Euclidean distance metric is the most commonly used for error measures in Neural Network applications. But it has been suggested that this distance metric is not appropriate for many problems [6]. In this work the aim is to find best error metric to use in Back propagation learning algorithm. The likelihood and log-likelihood functions are the basis for deriving estimators for parameters, for given set of data. In maximum likelihood method we estimate the value of ' $y$ ' for the given value of ' $x$ ' in presence of error. (See equation (1))

$y_{i}=x_{i}+e$ 
Let $x_{i}$ denote the data points in the distribution and let $N$ denotes the number of data points. Then an estimator $\bar{\mu}$ of $\mu$ can be estimated by minimizing the error metric with respect to $\bar{\mu}$.

$\varepsilon=\sum_{i=1}^{N} f(x, \bar{\mu})$.

Where $f(x, \bar{\mu})$ is distance metric.

Jie, et. al., [7-8] has proposed some new distance metrics based on different means. These distance metrics can be used to improve the performance of the neuron model for learning the best-fit weights of the neuron models. Distance metrics associated with the distribution models that imply the arithmetic mean, harmonic mean and geometric mean in (See Table 1) are inferred using equation

$\frac{d \varepsilon}{d \bar{\mu}}=\frac{d}{d \bar{\mu}} \sum_{i=1}^{N} f(x, \bar{\mu})=0$

In past, it is found that in the distribution associated with the harmonic and geometric estimations, the observations $x_{i}$ which are far away from $\bar{\mu}$ will contribute less towards $\mu$, in contrast to arithmetic mean and thus the estimated values will be less sensitive to the bad observations (i.e., observation with large variance), and therefore they are more robust in nature [9].

\begin{tabular}{|l|l|l|}
\multicolumn{1}{|l}{ Table 1 Error Metric Types and Mean } \\
\hline Arithmetic & Error metric & Mean \\
& $\varepsilon=\sum_{i=1}^{N}\left(x_{i}-\mu\right)^{2}$ & $\mu=\frac{1}{N} \sum_{i=1}^{N} x_{i}$ \\
\hline Harmonic & $\varepsilon=\sum_{i=1}^{N} x_{i}\left(\frac{\mu}{x_{i}}-1\right)^{2}$ & $\mu=\frac{N}{\sum_{i=1}^{N} x_{i}}$ \\
\hline Geometric & $\varepsilon=\sum_{i=1}^{N}\left[\log \left(\frac{\mu}{x_{i}}\right)\right]^{2}$ & $\mu=\frac{1}{N}\left(\Pi_{i=1}^{N} x_{i}\right)^{1 / N}$ \\
& & \\
\hline
\end{tabular}

Considering three layer structure of ANN, It has $n_{i}, n_{h}$ and $n_{o}$ neurons in input, hidden and output later respectively. Le the input and output vectors are $X=\left[x_{1}, x_{2}, \ldots x_{n i}\right]^{T}$ and $Y=\left[y_{1}, y_{2}, \ldots y_{n i}\right]^{T}$ respectively. Considering that the weight of the neuron that connects the $i^{\text {th }}$ neuron of input layer to $j^{\text {th }}$ neuron of output layer is $w_{i j}$ and bias of the

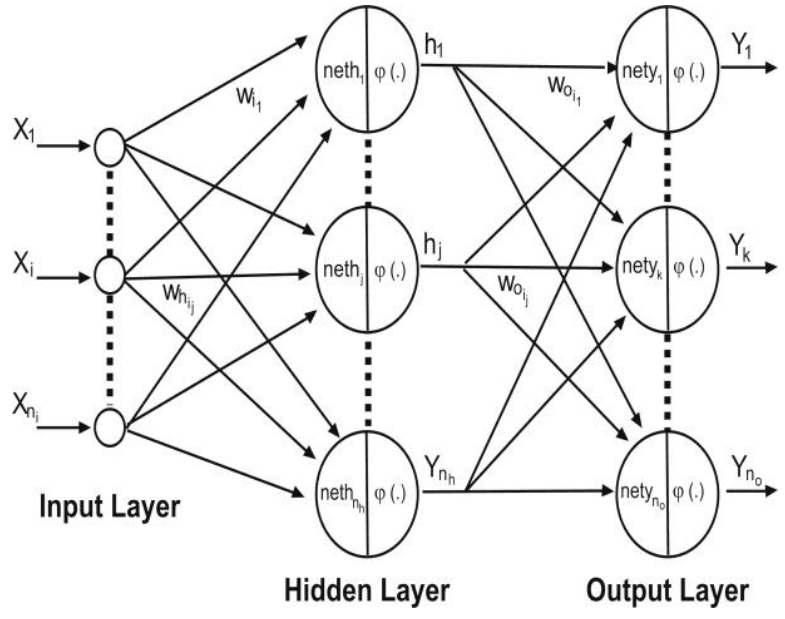

Figure 2 The Multilayer ANN Structure

$j^{\text {th }}$ neuron of the hidden layer is $b h_{j}$, the net value of the $j^{\text {th }}$ neuron is given by

neth $_{j}=\sum_{i=1}^{n_{i}} w h_{i j} \cdot x_{i}+b h_{j}$ where, $j=1,2 \ldots n_{h}$.

The output of the $j^{\text {th }}$ neuron $h_{j}$ of the hidden layer after applying the activation is defined as

$h_{j}=\varphi\left(\right.$ neth $\left._{j}\right)=\frac{1}{1+e^{- \text {neth }_{j}}}$.

Similarly, the net value nety $_{k}$ and the final output $y_{k}$ of the $k^{\text {th }}$ neuron of the output layer can be defined as

nety $_{k}=\sum_{i=1}^{n_{i}} w o_{i j} \cdot h_{j}+b h_{k}$ where, $k=1,2 \ldots n_{o}$.

The output of the $k^{\text {th }}$ neuron of the output layer

$y_{k}=\varphi\left(\right.$ nety $\left._{k}\right)=\frac{1}{1+e^{- \text {nety }_{k}}}$

In this section the error back-propagation learning of MLP with different error metrics have been derived. Let $\mathrm{E}$ denote the cumulative error at the output layer. In BP algorithm aim is to minimize the error at the output layer. The weight update equations using gradient descent rule are given bellow:

$$
\begin{aligned}
& w h_{j i}(n)=w h_{j i}(o)+\eta \frac{\partial E}{\partial y_{k}} \cdot \frac{\partial y_{k}}{\partial w h_{j i}} \\
& b h_{j}(n)=b h_{j}(o)+\eta \frac{\partial E}{\partial y_{k}} \cdot \frac{\partial y_{k}}{\partial b h_{j}} \\
& w o_{k j}(n)=w o_{k j}(o)+\eta \frac{\partial E}{\partial y_{k}} \cdot \frac{\partial y_{k}}{\partial w o_{k j}} \\
& b o_{k}(n)=b o_{k}(o)+\eta \frac{\partial E}{\partial y_{k}} \cdot \frac{\partial y_{k}}{\partial b k_{k}}
\end{aligned}
$$

Where, $\eta$ is learning rate and 


$$
\begin{aligned}
\frac{\partial y_{k}}{\partial w h_{j i}}=\left[\sum_{k=1}^{n_{0}}\left(1-y_{k}\right) \cdot y_{k} \cdot w_{k j}\right] \cdot\left(1-h_{j}\right) \cdot h_{j} \cdot x_{i} \\
\frac{\partial y_{k}}{\partial b h_{j}}=\left[\sum_{k=1}^{n_{0}}\left(1-y_{k}\right) \cdot y_{k} \cdot w_{k j}\right] \cdot\left(1-h_{j}\right) \cdot h_{j} \\
\frac{\partial y_{k}}{\partial b o_{k}}=\left[\sum_{k=1}^{n_{0}}\left(1-y_{k}\right) \cdot y_{k}\right] \\
\frac{\partial y_{k}}{\partial w o_{j k}}=\left[\sum_{k=1}^{n_{0}}\left(1-y_{k}\right) \cdot y_{k} \cdot h_{j}\right]
\end{aligned}
$$

For different error criterion only $\frac{\partial E}{\partial y}$ will change and is

shown as follows: This is dependent on the distance metric used in computation of the total error E.

\section{Case 1 Least Mean Square Error}

$L M S E=E=\frac{1}{2} \sum_{n=1}^{N} \sum_{k=1}^{n_{o}}\left(t_{k}-y_{k}\right)^{2}$, then

$\frac{\partial E}{\partial y_{k}}=-\eta\left(t_{k}-y_{k}\right)$

\section{Case 2 Geometric Error Metric}

$$
\begin{aligned}
& L M S E=E=\frac{1}{2} \sum_{n=1}^{N} \sum_{k=1}^{n_{o}} \log \left(\frac{y_{k}}{t_{k}}\right)^{2}, \text { then } \\
& \frac{\partial E}{\partial y_{k}}=-\eta\left(\log \left(t_{k}\right)-\log \left(y_{k}\right) \cdot \frac{1}{y_{k}}\right.
\end{aligned}
$$

\section{Case 3 Harmonic Error Metric}

$$
\begin{aligned}
& L M S E=E=\frac{1}{2} \sum_{n=1}^{N} \sum_{k=1}^{n_{o}}\left(\frac{y_{k}}{t_{k}}-1\right)^{2} \text {, then } \\
& \frac{\partial E}{\partial y_{k}}=-\eta \cdot t_{k} \cdot\left(\frac{y_{k}}{t_{k}}-1\right)
\end{aligned}
$$

Where, $y_{t}$ denotes the desired value of neuron and $t_{i}$ denotes the targeted value of the $i^{\text {th }}$ pattern.

\section{RESULTS}

In our experiment 421 words are considered (appendix A). These words are classified into seven groups. The group division is done in the broad categories: adult, financial, commercial, beauty and diet, travelling, home based and gambling. The ANN network is trained with 1200 words. Then the ANN used as classifier with different error metric presented in Table1. The correct classification data in percentage is shown in Table 2. It can be observed for the table that, arithmetic error type produces $96 \%$ accurate results while geometric error type produces $98 \%$ accurate results. Among the three, harmonic error metric produces $99 \%$ correct results.
Table 2: Percentage Classification under Various Error Matrixes.

\begin{tabular}{|l|l|}
\hline Type & Correct classification in percentage \\
\hline Arithmetic & $96 \%$ \\
\hline Geometric & $98 \%$ \\
\hline Harmonic & $99 \%$ \\
\hline
\end{tabular}

\section{CONCLUSIONS}

In this paper, ANN based data classifier is detailed with new error metric. Here, the wordlist we call it as data dictionary 421 words are considered as we use ANN based approach to classify them into seven categories. It has been fund that harmonic error metric data classification accuracy is $99 \%$.

\section{REFERENCES}

[1] S. Haykin, Neural Networks: A Comprehensive Foundation, MacMillan College Publishing Company, New York, 1995.

[2] Kanellopoulos, I., Varfiss, A., Wilkinson, G.G. and Megier, J., 1991: Neural network classification of multidata satelite imagery. Proceedings of International Geoscience and Remote Sensing Symposium (IGARSS'91), Espoo, Finland, June, pp. 2215-2218.

[3] Rich Drewes "An artificial neural network spam classifier", Project home page: www.interstice.com/drewes/cs676/spam-nn

[4] Sexton, S.R., Dorsey, R.E., "Reliable classification using neural networks: a genetic algorithm and backpropagation comparison", Decision Support Systems, 30: 11-22 (2000).

[5] Rumelhart, D.E., Hinton, G., Williams, R., "Learning representation by back-propagation errors", Nature, 323(9): 533-536 (1986).

[6] W. J. J. Rey, "Introduction to Robust and Quasi-Robust Statistical Methods", Springer-Verlag, Berlin, 110-116, 1983.

[7] J. Yu, J. Amores, N. Sebe, Q. Tian, "Toward an improve Error metric", International Conference on Image Processing (ICIP), 2004.

[8] J. Yu, J. Amores, N. Sebe, Q. Tian, "Toward Robust Distance Metric Analysis for Similarity Estimation", IEEE Computer Society Conference on Computer Vision and Pattern Recognition, 2006.

[9] [9] R. Battiti, "First and second-order methods for learning: between steepest descent and Newton's method", Neural Computation, Vol. 2, 141-166, 1992. 


\begin{tabular}{|c|c|c|}
\hline Group & Content & Example of Keywords in Each Group \\
\hline $\mathrm{C} 1$ & Adult & $\begin{array}{l}\text { adult, aphrodisiac, big, cam, climax, company, cum, desire, erotic, fantasy, fuck, gay, girl, } \\
\text { greate, guy, hard, hardcore, heaven, hot, huge, long, man, max, maxlength, nude, orgasm, } \\
\text { penis, performance, pheromone, pill, porn, powerful, pussy, satisfy, sex, stamina, sweet, } \\
\text { teen, viagra, webcam, x, xxx, xxx-porn, young, love, teen, anus }\end{array}$ \\
\hline $\mathrm{C} 2$ & Financial & $\begin{array}{l}\text { Account, accountant, alert, analyst, attorney, bank, bankruptcy, benefit, bill, billing, broker, } \\
\text { budget, building, cash, cheque, commission, consolidate, court, credit, creditor, currency, } \\
\text { customer, debt, deposit, discover, economy, entrepreneur, estate, exchange, fee, finance, } \\
\text { freedom, fund, help, high-risk, insurance, invest, } \\
\text { investor, judgment, legal, legitimate, lender, loan, mastercard, mortgage, obligate, pay, } \\
\text { payable, payable, paycheck, promote, purchase, rate, refinance, refund, rent, revenue, risk, } \\
\text { service, statement, stock, support, tax, transaction, vat, visa, wealth, worth, service }\end{array}$ \\
\hline $\mathrm{C} 3$ & Commercial & $\begin{array}{l}\text { college, commerce, computer, cost, deliver, discount, especial, expensive, express, } \\
\text { fantastic, free, furnishing, furniture, game, get, gif, gift, great, guarantee, inexpensive, } \\
\text { invite, item, just, keyboard, license, lifetime, magazine, maintenance, mall, market, } \\
\text { material, materials, mobile, motherboard, mouse, offer, online, only, } \\
\text { order, palm, pamphlet, percent, premium, price, produce, product, program, recommend, } \\
\text { refill, release, resell, reseller, retail, sale, save, save, sell, ship, shipping, shop, shopping, } \\
\text { special, subscribe, supply, surprise, trade, trademark, upgrade, voucher, whole, wholesale, } \\
\text { within }\end{array}$ \\
\hline $\mathrm{C} 4$ & $\begin{array}{l}\text { Beauty and } \\
\text { Diet }\end{array}$ & $\begin{array}{l}\text { after, age, amaze, anti-aging, appetite, beauty, become, before, believe, blood, body, } \\
\text { botanic, breast, build, burn, Diet calorie, capsule, card, cell, change, chemical, cholesterol, } \\
\text { confirm, course, diet, difference, dose, drug, effect, effective, eliminate, energy, enhance, } \\
\text { exercise, eye, face, fast, fat, firm, fit, fitness, flexible, gary, grow, grown, growth, hair, } \\
\text { health, healthcare, heart, height, herb, herbal, hormone, improve, inche, incredible, kidney, } \\
\text { large, } \\
\text { laser, life-changing, light, lose, loss, low, magic, medicine, metabolism, micro-cap, miracle, } \\
\text { modem, move, muscle, nature, nutrient, old, over, overweight, permanent, plain, potential, } \\
\text { pound, power, protect, reduce, remanufacture, repair, restore, retain, reverse, safe, } \\
\text { satisfaction, secret, size, step, strength, strong, tablet, } \\
\text { therapy, thin, toxin, treatment, under, virginia, vitamin, weight, woman, wonderful, wrinkle }\end{array}$ \\
\hline $\mathrm{C} 5$ & Traveling & $\begin{array}{l}\text { book, deluxe, excite, guide, holiday, honest, hotel, luxury, meal, package, plan, problem, } \\
\text { relax, relief, reserve, resort, summer, temple, ticket, tour, train, travel, traveler, trip, } \\
\text { vacation, }\end{array}$ \\
\hline C 6 & Home-Based & $\begin{array}{l}\text { address, astonishment, base, broadcast, bulk, business, comfort, connect, demo, domain, } \\
\text { downline, download, Business earn, email, emailing, ethernet, facemail, fresh, home, } \\
\text { homebased, homeworker, host, income, interest, international, internet, investigate, job, list, } \\
\text { lucrative, mail, mailbox, mailer, mailing, make, marketing, message, million, money- } \\
\text { making, opportunity, part-time, people, private, profit, reach, receive, recipient, require, re- } \\
\text { register, return, server, software, subscriber, success, teach, unsubscribe, user, visit, } \\
\text { website, work, work-at-home, worker, working }\end{array}$ \\
\hline $\mathrm{C} 7$ & Gambling & $\begin{array}{l}\text { action, award, bet, bonus, casino, challenge, extra, gambling, gold, hunt, las, lucky, } \\
\text { millionaire, player, poker, prize, reward, rich, vegas, win, lottery }\end{array}$ \\
\hline
\end{tabular}

